\title{
A resonance theory consistent with Mulliken-population concept
}

\section{$\operatorname{AUTHOR}(\mathrm{S})$ :}

Ikeda, Atsushi; Nakao, Yoshihide; Sato, Hirofumi; Sakaki, Shigeyoshi

\section{CITATION:}

Ikeda, Atsushi ...[et al]. A resonance theory consistent with Mullikenpopulation concept. Chemical Physics Letters 2011, 505(4-6): 148-153

\section{ISSUE DATE:}

2011-03

URL:

http://hdl.handle.net/2433/158036

\section{RIGHT:}

C 2011 Elsevier B.V.; この論文は出版社版でありません。引用の際には 出版社版をご確認ご利用ください。; This is not the published version. Please cite only the published version. 


\title{
A Resonance Theory Consistent with Mulliken Population
}

\author{
Atsushi Ikeda ${ }^{1}$, Yoshihide Nakao, Hirofumi Sato*, Shigeyoshi Sakaki ${ }^{2}$ \\ Department of Molecular Engineering, Graduate School of Engineering, Kyoto University, Nishikyo-ku, Kyoto 615-8510, Japan
}

\begin{abstract}
An analysis of molecular orbital wave function from the viewpoint of resonance theory is proposed. This is an extension of our previous method to treat conjugated-electron system. It enables us to calculate the weights of resonance structures being consistent with Mulliken population as well as bond order analysis. The method is successfully applied to LiH, $\mathrm{H}_{2} \mathrm{O}$, butadiene and $\mathrm{S}_{\mathrm{N}} 2$ reaction.
\end{abstract}

Keywords:

resonance structure, molecular orbital, valence bond, Mulliken population, bond order,

\section{Introduction}

Chemist's traditional approach to molecule is based on the resonance theory. In the theory, the electronic structure of molecule is understood in terms of resonance between several resonance structures, which are built of atoms linking each other through ionic and/or covalent bond. Linus Pauling describes the electronic structure of $\mathrm{HCl}$ in his textbook "The chemical bond" as follows;[1]

For a molecule such as hydrogen chloride we write the two reasonable electronic structures $\mathrm{H}: \ddot{\mathrm{C}} \mathrm{l}$ : and $\mathrm{H}^{+}: \ddot{\mathrm{C}}:^{-}$. (The third structure that suggests itself, $\mathrm{H}^{-} \mathrm{Cl}^{+}$, is not given much importance because hydrogen is recognized as less electronegative than chlorine ...) ... In accordance with the foregoing argument the actual state of the molecule can be described as corresponding to resonance between these two structures.

We may describe the bond as a covalent bond with partial ionic character, and make use of the valence line, writing $\mathrm{H}-\mathrm{Cl}$ (or $\mathrm{H}-\ddot{C} \mathrm{l}:$ ) in place of $\{\mathrm{H}: \ddot{\mathrm{C}} \mathrm{l}$ : , $\left.\mathrm{H}^{+} \mathrm{Cl}^{-}\right\}$or some similar complex symbol showing resonance between the two extremes.

The view is still useful to provide simple understanding of the reactivity and character of molecule. However, most of modern ab-initio calculations are based on the molecular orbital (MO) theory and it is hard to extract the traditional picture of molecule from a MO wave function. Because of these factors,

\footnotetext{
${ }^{*}$ Corresponding author

Email address: hirof umi@moleng.kyoto-u.ac.jp (Hirofumi Sato)

${ }^{1}$ Present address: Institute of Physics, University of Tsukuba, Tennodai 11-1, Tsukuba 305-8571, Japan

${ }^{2}$ Present address: Institute for Integrated Cell-Material Sciences, Kyoto University, Yoshida Ushinomiya-cho, Sakyo-ku, Kyoto 606-8501, Japan
}

development of analysis for MO wave function is highly desired to elucidate the nature of chemical bonding. For such purpose, Hiberty et al.[2] reported the method to calculate the weights of resonance structures, in which MO wave function is expanded into a set of valence-bond (VB) type wave functions. Weinhold et al. reported quite different method, called natural resonance theory (NRT) based on natural bond orbital (NBO) analysis.[3] Karafiloglou et al. are working vigorously to address this problem[4-7] and other several methods have been developed including works by Shaik et al.[8], CASVB by Robb et al.[9] and the method based on CASSCF-type wave function by Hirao and coworkers[10]. MOVB by Mo and Gao is one of direct realizations that fit the present purpose[11-14]. Another type of analysis based on locally defined energy by Nakai et al. can also offer detailed inside of the electronic structure of a molecule and its bonds.[15-24]

Several types of analysis methods have been traditionally developed. Mulliken population analysis (MPA) and ab-initio bond order analysis (BOA) are the representative. A theoretical background of MPA became very clear by Mayer's reformulation using mixed formalism of the second quantization for nonorthogonal orbitals[25]. BOA was independently developed by Mayer[25, 26], Giambiag et al.[27-29] and by Okada et al.[30, 31] as an extension of Wiberg's pioneering work[32]. Furthermore, several definitions, interpretations, and extensions of atomic population and bond order have been developed by various researchers[33-36].

We recently reported a method to evaluate the weights of resonance structures from Hartree-Fock (HF) wave function, which is based on an orbital localization and a second quantized expression.[37-40] The results show excellent agreement with our chemical intuitions as well as with previous computations. However, the applicability of the method was limited to the system in which electrons are strictly localized. For this reason, an electron-conjugated molecular system including the transition states of reactions could not be treated. In this work, we developed a novel method to calculate the weights of resonance 
structures easily applicable to a conjugated system. Although the present method allows us to treat not over four conjugating electrons, it significantly expands the versatility of the analysis as demonstrated below.

Another important feature of the present method is the consistency with MPA and BOA. Let us consider $\mathrm{H}_{2}$ as a trivial example. According to the above mentioned Pauling's picture, there are three resonance structures, namely, $\mathrm{H}_{a}-\mathrm{H}_{b}, \mathrm{H}_{a}^{-} \mathrm{H}_{b}^{+}$ and $\mathrm{H}_{a}^{+} \mathrm{H}_{b}^{-}$(labeled $R=1,2,3$, respectively). Note that the formal charge assigned to atom $\mathrm{A}\left(\left\{Q_{R}^{\mathrm{A}}\right\}\right)$ and formal bond order between $\mathrm{A}$ and $\mathrm{B}\left(\left\{\mathcal{B}_{R}^{\mathrm{A}-\mathrm{B}}\right\}\right)$ are always integer in this framework. In the case of $\mathrm{H}_{a}-\mathrm{H}_{b}$, the formal charges assigned to $\mathrm{H}_{a}$ $\left(Q_{1}^{\mathrm{a}}\right)$ and $\mathrm{H}_{b}\left(Q_{1}^{\mathrm{b}}\right)$ are respectively 1 , and the formal bond order $\left(\mathcal{B}_{1}^{\mathrm{a}-\mathrm{b}}\right)$ is 1 . In a similar manner, an ionic structure $\left(\mathrm{H}_{a}^{-} \mathrm{H}_{b}^{+}\right)$, the charge to $\mathrm{H}_{a}$ and $\mathrm{H}_{b}$ are respectively $2\left(=Q_{2}^{\mathrm{a}}\right)$ and $0\left(=Q_{2}^{\mathrm{b}}\right)$ with the formal bond order of $0\left(=\mathcal{B}_{2}^{\mathrm{a}-\mathrm{b}}\right)$. And basically the same for $\mathrm{H}_{a}^{+} \mathrm{H}_{b}^{-}$. According to Pauling, the bond is described as involving resonance between these extreme bond-pictures with some amount (weight) of each contribution. Here we consider weight $\left(W_{R}\right)$ of each resonance structure that satisfies the following equations.

$$
\begin{aligned}
\sum_{R} W_{R} & \times Q_{R}^{\mathrm{A}} \\
& =\text { Mulliken population on atom } \mathrm{A} \\
2 \sum_{R} W_{R} & \times \mathcal{B}_{R}^{\mathrm{A}-\mathrm{B}} \\
& =\text { Bond order between } \mathrm{A} \text { and } \mathrm{B} \text { atoms }
\end{aligned}
$$

The aim of the present study is to seek $W_{R}$ that exactly satisfies these equations and here we present such an expression in four electron system computed by RHF method.

The organization of this paper is follows. In Sec 2, we overview our previous method and formulated the new method. In $\mathrm{Sec} 3$, the new method is applied to $\mathrm{LiH}$ and $\mathrm{H}_{2} \mathrm{O}$ molecules, to investigate the consistency with MPA, BOA and our previous method. Next, trans-butadiene and $\mathrm{S}_{\mathrm{N}} 2$ reaction are examined. Both of them can not be treated by the previous method because of the electron delocalization. The conclusion was summarized in Sec 4.

\section{Theory}

\subsection{A reformulation of $B O A$}

$\mathrm{Ab}$-initio partial bond order between atomic orbitals $a$ and $b$ can be defined as follows,

$$
b_{a: b}^{1}=\frac{1}{2}(\mathbf{P S})_{a b}(\mathbf{P S})_{b a}
$$

Here, $\mathbf{S}$ is overlap matrix and $\mathbf{P}$ is defined as $P_{a b}=$ $2 \sum_{i}^{o c c} C_{a i} C_{b i}^{*}$, where $C_{a i}$ is LCAO coefficient of molecular orbital $i$. The bond order quantity between atoms $\mathrm{A}$ and $\mathrm{B}$, known as the Mayer bond index, [25, 26] is introduced by Giambiagi et al. [27-29] as well as by Okada et al.[30, 31], which is an extension of Wiberg's work [32].

$$
B_{A B}=2 \sum_{a \in A} \sum_{b \in B} b_{a: b}^{1}=\sum_{a \in \mathrm{A}} \sum_{b \in \mathrm{B}}(\mathbf{P S})_{a b}(\mathbf{P S})_{b a} .
$$

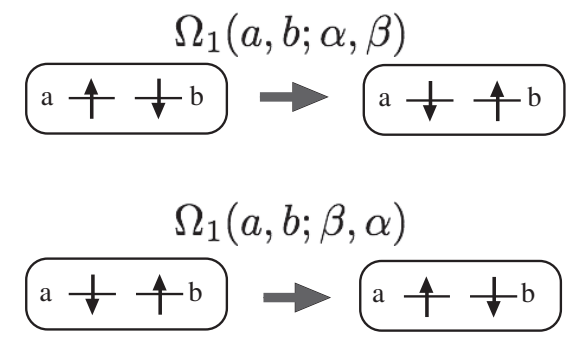

Figure 1: Operation of $\Omega_{1}(a, b ; \alpha, \beta)$ and $\Omega_{1}(a, b ; \beta, \alpha)$.

The partial bond order is also described as an expectation value of wave function $\left(\Psi_{\mathrm{HF}}\right)$ using the second quantized operator.

$$
\begin{aligned}
b_{a: b}^{1} & =\left\langle\Psi_{\mathrm{HF}}\left|\chi_{b}^{\alpha+} \chi_{a}^{\beta+} \phi_{b}^{\beta-} \phi_{a}^{\alpha-}+\chi_{b}^{\beta+} \chi_{a}^{\alpha+} \phi_{b}^{\alpha-} \phi_{a}^{\beta-}\right| \Psi_{\mathrm{HF}}\right\rangle \\
& =\left\langle\Psi_{\mathrm{HF}}\left|\Omega_{1}(a, b ; \alpha, \beta)+\Omega_{1}(a, b ; \beta, \alpha)\right| \Psi_{\mathrm{HF}}\right\rangle
\end{aligned}
$$

where the following operator is introduced.

$$
\Omega_{1}\left(m, n ; \sigma_{m}, \sigma_{n}\right)=\chi_{n}^{\overline{\sigma_{n}}+} \chi_{m}^{\overline{\sigma_{m}}+} \phi_{n}^{\sigma_{n}-} \phi_{m}^{\sigma_{m}-} .
$$

Here, $\chi_{m}^{+}$is creation operator of nonorthogonal atomic orbital $m$ and $\phi_{m}^{-}$is annihilation operator of biorthogonal atomic orbital $m$. $\overline{\sigma_{m}}$ means the opposite spin of $\sigma_{m}$. When and only when the ket is the states in which two atomic orbitals $a$ and $b$ are singly occupied by one $\alpha$ electron and one $\beta$ electron, the operators $\Omega_{1}(a, b ; \alpha, \beta)$ and $\Omega_{1}(a, b ; \beta, \alpha)$ flip $\alpha$ and $\beta$ spin into $\beta$ and $\alpha$, respectively (Figure 1), indicating that $b_{a: b}^{1}$ corresponds to the probability of the state in which two electrons are singletcoupled between atomic orbitals. The present work deals with closed-shell RHF wave function and the operator properly characterizes molecular electronic structure as shown in the previous works[37-40], namely, the contribution from triplet does not contaminate the state. Note that there is close correspondence between the present operator $\Omega_{1}$ and the spin-flip operator introduced by Clark and Davidson[41-43], whose aim is deeply related to the present work. More extensive investigation and in-depth comparison with the their operator is significant for generalization including high-spin state, although it is beyond the scope of the present study. Because the spincoordinate is attached to the spatial function in the present treatment (biorthogonalized orbital based on second quantization), it is not trivial to apply a simple analogy to the singlet-coupling in orthogonal orbital.

\subsection{The previous method}

Let us consider a localized orbital $\psi_{i}$ between two atomic centers, $\mathrm{X}$ and $\mathrm{Y}$, that is obtained by standard orbital localization procedure. In the previous work [37-40], we found that the weights of ionicity and covalency are obtained by,

$$
\begin{aligned}
W^{i}\left(\mathrm{X}^{-} \mathrm{Y}^{+}\right) & =\frac{1}{2} \sum_{a \in X} \sum_{b \in X} b_{a: b}^{1(i)}, \\
W^{i}(\mathrm{X}-\mathrm{Y}) & =\sum_{a \in X} \sum_{b \in Y} b_{a: b}^{1(i)}, \\
W^{i}\left(\mathrm{X}^{+} \mathrm{Y}^{-}\right) & =\frac{1}{2} \sum_{a \in Y} \sum_{b \in Y} b_{a: b}^{1(i)},
\end{aligned}
$$


where

$$
b_{a: b}^{1(i)}=\left\langle\psi_{i} \bar{\psi}_{i}\left|\Omega_{1}(a, b ; \alpha, \beta)+\Omega_{1}(a, b ; \beta, \alpha)\right| \psi_{i} \bar{\psi}_{i}\right\rangle .
$$

Here the same operator was employed, but the essential difference from the above equation is that it is applied only to the localized orbital $\left(\psi_{i}\right)$ related to the atomic orbitals $a$ and $b$. The localization is essential to eliminate the contribution from orbitals (and/or electrons) that do not participate the bond formation. As described in the previous works, the weights of resonance structures are properly computed using this operator, $b_{a: b}^{1(i)}$.

We would like to stress that our approach is essentially different from previous works. It would be a standard manner to re-expand $\left|\Psi_{\mathrm{HF}}\right\rangle$ in terms of different set of (VB) wave function. Our strategy is to expand the identity operator so as to makes chemical intuitive sense (For the detail, please refer the previous works[37, 40]). In NRT, density-averaging (resonanceaveraging) is central assumption, which might be completed without quantum effect. In our treatment, the density distribution is derived through the weight related to the resonance structure as a consequence of calculation of an expectation value of wave function. It would also be valuable to point out the quantity seen in a different way. For example,

$$
\left\langle\Psi\left|\Omega_{1}\left(m, n ; \sigma_{m}, \sigma_{n}\right)\right| \Psi\right\rangle=\left\langle\Psi\left|\chi_{n}^{\overline{\sigma_{n}+}} \chi_{m}^{\overline{\sigma_{m}}+} \cdot \phi_{n}^{\sigma_{n}-} \phi_{m}^{\sigma_{m}-}\right| \Psi\right\rangle .
$$

Since the biorthogonal operator is employed, the bra part $\left(\langle\Psi| \chi_{n}^{\overline{\sigma_{n}}+} \chi_{m}^{\overline{\sigma_{m}}+}\right)$ and the remaining ket part is slightly different but essentially equivalent.

\subsection{An extension of the method}

In the previous method, each pair of electrons is assumed to be localized in one chemical bond $\left(\psi_{i}\right)$. The assumption may be invalid when considering the delocalized electron, for instance, conjugated molecular system and transition states of chemical reaction. A simple strategy to overcome difficulty is to define the 'second-order' bond order $b_{a: b, c: d}^{2}$, which is related to the probability of the state in which four electrons make two singlet-coupled pairs, namely between $a$ and $b$, and between $c$ and $d$. By extending the operators in Eq. (5), the following operator is introduced.

$$
\begin{array}{r}
\Omega_{2}\left(a, b, c, d ; \sigma_{a}, \sigma_{b}, \sigma_{c}, \sigma_{d}\right) \equiv \Omega_{2}\left(\sigma_{a}, \sigma_{b}, \sigma_{c}, \sigma_{d}\right) \\
=\chi_{d}^{\sigma_{d}+} \chi_{c}^{\sigma_{c}+} \chi_{b}^{\sigma_{b}+} \chi_{a}^{\sigma_{a}+} \phi_{d}^{\sigma_{d}-} \phi_{c}^{\sigma_{c}-} \phi_{b}^{\sigma_{b}-} \phi_{a}^{\sigma_{a}-} .
\end{array}
$$

For the sake of simplicity, the indices, $a, b, c, d$, are omitted. Similar to $\Omega_{1}$, the operator flips $\alpha$ and $\beta$ spin into $\beta$ and $\alpha$ electrons, respectively. By considering all the possible combinations of electron pair, the second-order bond index $b_{a: b, c: d}^{2}$ is defined as follows,

$$
\begin{aligned}
b_{a: b, c: d}^{2} & =\langle\Psi| \Omega_{2}(\alpha, \beta, \alpha, \beta)+\Omega_{2}(\alpha, \beta, \beta, \alpha) \\
& +\Omega_{2}(\beta, \alpha, \alpha, \beta)+\Omega_{2}(\alpha, \beta, \beta, \alpha) \\
& -\Omega_{2}(\alpha, \alpha, \beta, \beta)-\Omega_{2}(\beta, \beta, \alpha, \alpha)|\Psi\rangle \\
& \equiv\langle\Psi|\Omega| \Psi\rangle .
\end{aligned}
$$

Since $a$ and $b(c$ and $d$ ) are bonded, respective spin on them never become parallel at the same time, which is represented in the last two terms with minus sign. For example, a different bonding situation, $b_{a: c, b: d}^{2}$, is written in a similar operator with different combination of signs,

$$
\begin{aligned}
\Omega= & -\Omega_{2}(a, b, c, d ; \alpha, \beta, \alpha, \beta) \\
& +\Omega_{2}(a, b, c, d ; \alpha, \beta, \beta, \alpha) \\
& +\Omega_{2}(a, b, c, d ; \beta, \alpha, \alpha, \beta) \\
& -\Omega_{2}(a, b, c, d ; \beta, \alpha, \beta, \alpha) \\
& +\Omega_{2}(a, b, c, d ; \beta, \beta, \alpha, \alpha) \\
& +\Omega_{2}(a, b, c, d ; \alpha, \alpha, \beta, \beta) .
\end{aligned}
$$

For the Hartree-Fock closed shell wave function, $b_{a: b, c: d}^{2}$ is simply expressed by one-body matrix element,

$$
\begin{aligned}
b_{a: b, c: d}^{2} & =\frac{1}{4}(\mathbf{P S})_{a b}(\mathbf{P S})_{b a}(\mathbf{P S})_{c d}(\mathbf{P S})_{d c} \\
& -\frac{1}{8}(\mathbf{P S})_{a c}(\mathbf{P S})_{c d}(\mathbf{P S})_{d b}(\mathbf{P S})_{b a} \\
& -\frac{1}{8}(\mathbf{P S})_{a b}(\mathbf{P S})_{b d}(\mathbf{P S})_{d c}(\mathbf{P S})_{c a} \\
& -\frac{1}{8}(\mathbf{P S})_{a b}(\mathbf{P S})_{b c}(\mathbf{P S})_{c d}(\mathbf{P S})_{d a} \\
& -\frac{1}{8}(\mathbf{P S})_{a d}(\mathbf{P S})_{d c}(\mathbf{P S})_{c b}(\mathbf{P S})_{b a} \\
& +\frac{1}{8}(\mathbf{P S})_{a c}(\mathbf{P S})_{c b}(\mathbf{P S})_{b d}(\mathbf{P S})_{d a} \\
& +\frac{1}{8}(\mathbf{P S})_{a d}(\mathbf{P S})_{d b}(\mathbf{P S})_{b c}(\mathbf{P S})_{c a}
\end{aligned}
$$

The operator and derived equation look complex at the first glance, but the term can be computed just as multiplication of PS matrix element. We found that this operator satisfactorily represents the situation of chemical bonds as shown below. It is much more important that the expression satisfies simple relationships. Summing up the index $c$ and $d$ immediately leads to,

$$
\begin{aligned}
\sum_{c} \sum_{d} b_{a: b, c: d}^{2} & =\left(\frac{N_{e}}{2}-1\right)(\mathbf{P S})_{a b}(\mathbf{P S})_{b a} \\
& =\left(\frac{N_{e}}{2}-1\right) 2 b_{a: b}^{1},
\end{aligned}
$$

using the duodempotency and the trace-conservation of PS,

$$
(\mathbf{P S})_{i j}=\frac{1}{2} \sum_{k}(\mathbf{P S})_{i k}(\mathbf{P S})_{k j}, \quad \text { and } \quad N_{e}=\sum_{k}(\mathbf{P S})_{k k},
$$

where $N_{e}$ is the number of electrons. The equation is related to the bond index $\left(b_{a: b}^{1}\right)$ by integration of all other electron-pairs in the system $\left(N_{e} / 2-1\right)$. By utilizing the well-known relationship between bond index and $N_{e}\left(\sum_{A B} B_{A B}=2 N_{e}\right)$, the further summing up gives,

$$
\begin{aligned}
\sum_{b} \sum_{c} \sum_{d} b_{a: b, c: d}^{2} & =\left(N_{e}-2\right) \sum_{b} b_{a: b}^{1} \\
& =\left(\frac{N_{e}}{2}-1\right) \cdot 2(\mathbf{P S})_{a a}, \\
\sum_{a} \sum_{b} \sum_{c} \sum_{d} b_{a: b, c: d}^{2} & =4 \cdot\left(\frac{N_{e}}{2}-1\right) \frac{N_{e}}{2} .
\end{aligned}
$$


or

$$
\frac{1}{4} \sum_{a} \sum_{b} \sum_{c} \sum_{d} b_{a: b, c: d}^{2}=\left(\frac{N_{e}}{2}-1\right) \frac{N_{e}}{2} .
$$

The facts implies that the definition of $b_{a: b, c: d}^{2}$ is the consist with MPA and popular BOA. As a matter of fact, the derived weights exactly satisfies Eqs. (1) and (2) for four electron systems. The proposed definition of $b_{a: b, c: d}^{2}$ also satisfies a requirement in corresponding VB wave function; Two covalent bonds must not use the same atomic orbitals at the same time. The corresponding quantity $b_{a: b, a: c}^{2}$ becomes zero by definition.

Now, we can compute the weights of resonance structures using this operator, $\Omega$. The above equations can be directly employed in four-electron systems such as $\mathrm{LiH}$, but not be limited. They are also applicable to analyze the electronic structure of 'sub-system' consisting of four electrons in general molecule. Let us consider two localized orbitals $\psi_{i}$ and $\psi_{j}$ in the vicinity of atomic centers, A, B, C, and D. The second-order ab-initio bond order related to these orbitals is introduced as follows.

$$
b_{a: b, c: d}^{2(i, j)}=\left\langle\psi_{i} \psi_{j}|\Omega| \psi_{i} \psi_{j}\right\rangle .
$$

By the same token, the procedure of localization is also useful to remove the contribution from one-center orbitals (core orbital, lone-pair orbital and so on) that do not participate the bond formation. For the details, please refer the previous works[37, 40]. Using density matrix computed from the localized orbitals, the weights of resonance structure is easily easily computed. For example, the weight, in which both of A-B and $\mathrm{C}-\mathrm{D}$ are covalent, can be expressed as follows,

$$
W^{i, j}(\mathrm{~A}-\mathrm{B}, \mathrm{C}-\mathrm{D})=\sum_{a \in A} \sum_{b \in B} \sum_{c \in C} \sum_{d \in D} b_{a: b, c: d}^{2(i, j)} \text {. }
$$

Similarly, the weight of a partially ionic structure, $\mathrm{A}^{-} \mathrm{B}^{+}$and $\mathrm{C}-\mathrm{D}$, is given by,

$$
W^{i, j}\left(\mathrm{~A}^{-} \mathrm{B}^{+}, \mathrm{C}-\mathrm{D}\right)=\sum_{a \in A} \sum_{b \in A} \sum_{c \in C} \sum_{d \in D} b_{a: b, c: d}^{2(i, j)},
$$

and so on. As we will see later, the calculated weights computed by this formula fits to our chemical intuition. Equations (1) and (2) are rigorously satisfied in the subspace occupied by four electrons, and guarantee the normalization of the weights. As shown below, the obtained results by this expression makes chemical intuitive sense. The expression is, on the other hand, limited to closed-shell with four-electron (sub) system and several problems remain to be solved to generalize the method so as to treat $N$-electron system. The electronic correlation effect might be evaluated by a simple procedure[45] or by direct consideration of many-body density but much more elaboration is required to take into account the correlation effect.

In the present work, all calculations were performed with program code GAMESS [46] modified by us. MO was obtained using the built-in basis sets, namely STO-3G, DZV, DZP and TZP (5d) at the respective optimized geometry. Pipek-Mezey orbital localization [47] scheme was then employed. Similar to the previous studies, [37-40] we found that the weight is virtually independent to the choice of localization scheme as well as to basis set.
Table 1: The weights (\%) of resonance structures, bond order, and atomic population of $\mathrm{LiH}$. Mayer's bond order and Mulliken population are also showed.

\begin{tabular}{ccccc}
\hline \hline No. & & DZV & DZP & TZP \\
\hline $\mathbf{1}$ & $\mathrm{Li}-\mathrm{H}$ & 46.9 & 48.4 & 44.0 \\
\cline { 3 - 5 } $\mathbf{2}$ & $\mathrm{Li}^{-} \mathrm{H}^{+}$ & 14.1 & 17.0 & 10.7 \\
\cline { 3 - 5 } $\mathbf{3}$ & $\mathrm{Li}^{+} \mathrm{H}^{-}$ & 39.1 & 34.6 & 45.3 \\
\hline Bond & Present $^{a}$ & 0.937 & 0.970 & 0.880 \\
order & BOA $^{a}$ & 0.937 & 0.970 & 0.880 \\
\hline $\mathrm{Li}$ & Present $^{a}$ & 2.750 & 2.823 & 2.654 \\
population & MPA & 2.750 & 2.823 & 2.654 \\
\hline $\mathrm{H}$ & Present $^{a}$ & 1.250 & 1.177 & 1.346 \\
population & MPA & 1.250 & 1.177 & 1.346 \\
\hline \hline
\end{tabular}

a) The values are calculated by using Eqs. 1 and 2 .

\section{Numerical results and discussion}

\subsection{LiH and $\mathrm{H}_{2} \mathrm{O}$ : Consistency with MPA, BOA and the previ- ous method.}

To demonstrate the consistency with MPA and BOA, we apply the present method to $\mathrm{LiH}$ molecule, as a typical four electron system. The weights of resonance structures, populations of $\mathrm{Li}$ and $\mathrm{H}$, and bond orders calculated by MPA, BOA, and the present method are shown in Table 1. The charges and bond orders calculated by the present method are exactly equal to MPA and BOA values in all the basis sets. The weights slightly depend on the choice of basis set, and this dependency is also consistent with MPA and BOA. For example, using the weights of $\mathrm{Li}^{+} \mathrm{H}^{-}(45.3 \%), \mathrm{Li}^{-} \mathrm{H}^{+}(10.7 \%)$ and $\mathrm{Li}-\mathrm{H}(44.0 \%)$ obtained by TZP and the formal values of electron number, population assigned to $\mathrm{Li}$ is calculated by Eq. (1).

$$
(2 \times 0.453)+(4 \times 0.107)+(3 \times 0.440)=2.654,
$$

namely, $3-2.654=+0.346$ is the charge derived from the weight computed by the present method, which is exactly the same as the Mulliken charge, by definition.

In the previous studies, the wave function of $\mathrm{H}_{2} \mathrm{O}$ was analyzed concerning the two $\mathrm{O}-\mathrm{H}$ bonds. Both of the previous and present methods were employed to calculate the weights as listed in Table 2. Weights computed by the two methods are very similar in all the basis sets, thought value by the present method tends to be slightly smaller than the previous one. This might be attributed to the difference in the possible number of resonance structure described in each method. In the previous method, nine structures (when counting degenerated structures separately; 1-6) shown in Table 2 are taken into account except for the many-body type contributions. Other type structure such as 7 can be treated only in the present method. Although this contribution is negligibly small and hence the computational weights by the two methods are very similar, this feature is one of the essential differences between the two methods, especially in the system where the electron delocalization is significant. In other words, the previous method independently treats two orbitals in $\mathrm{H}_{2} \mathrm{O}$, while the present one directly considers two pairs of electrons (four electrons) in valence orbitals. Since the 
Table 2: The weights (\%) of resonance structure in $\mathrm{H}_{2} \mathrm{O}$ calculated by the previous and present methods.

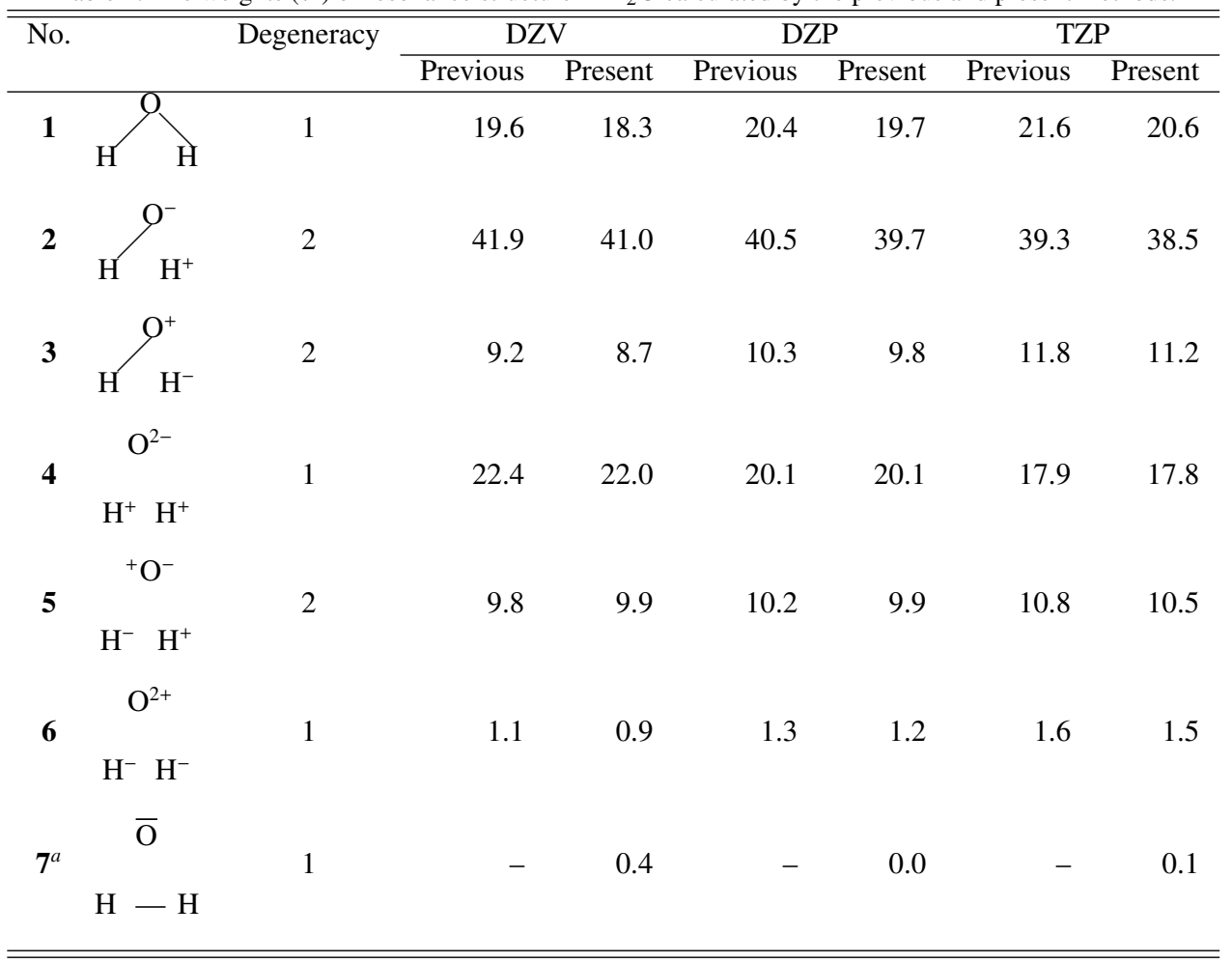

a) Two hydrogen atoms are covalently bonded and a remaining pair of electrons is in the oxygen atom. The contribution is not available with the previous method.

weight used here is an analogue of MPA, this weight, in principle, can be negative or larger than one as already shown in our previous work[40]. In practice, it poses little problem for understanding of the bonding nature.

\section{2. trans-butadiene}

In this section, we focus on trans-butadiene, in which the electron is inherently delocalized. Hiberty et al. calculated the weights of resonance structure using their method, in which the MO wave function is expanded into VB type of wave functions, with minimal basis sets[45]. The method is reliable but difficult to apply with extended basis sets, because VB type of wave function should be defined by minimal-type (or hybrid) orbital. On the other hand, the present method is easy to apply with extended basis sets. The weights of resonance structures computed by the present method are summarized in Table 3. Various basis sets were employed by selecting the four conjugated electrons in two $\pi$ orbitals.

The present results are very similar to the Hiberty's one. Note that small numerical deviation could be caused by the difference in employed geometry (corresponding to experimental geometry and optimized one) and essentially the same results are obtained. Major contributing resonance structures are 1, 3 and 4, which contain terminus double bonds. To the contrary, the weights of the structures with the central double bond, $\mathbf{2}$ and $\mathbf{5}$, are very small. It is also noted that the present method is virtu- ally independent to the basis sets as seen in the table. The same trend was also seen in the previous method.[40]

\section{3. $S_{\mathrm{N}} 2$ reaction}

Finally, we applied the method to $\mathrm{S}_{\mathrm{N}} 2$ reaction, $\mathrm{Cl}_{b}^{-}+\mathrm{CH}_{3} \mathrm{Cl}_{a}$ $\rightarrow \mathrm{Cl}_{b} \mathrm{CH}_{3}+\mathrm{Cl}_{a}^{-}$. Because four electrons related to bonds breaking and formation are delocalized especially in the transition state, the previous method cannot be applied to this system. The reaction coordinate is selected as $R_{1}-R_{2}$, where $R_{1}$ is the distance between $\mathrm{C}$ and $\mathrm{Cl}_{a}, R_{2}$ is between $\mathrm{C}$ and $\mathrm{Cl}_{b}$, as shown in Figure 2. The other degrees of freedom were fully optimized using TZP basis set, then two localized orbitals related to the aforementioned four electrons that participate the reaction were chosen. The weights of resonance structures in the transition state is shown in Table 4. The most important resonance structure is $\mathbf{2}$ which is the totally ionic $(63.0 \%)$. The next is $\mathbf{1}$ and 3 , in which one $\mathrm{Cl}$ becomes an ion and the other $\mathrm{Cl}$ is covalently bonded to $\mathrm{C}(18.5 \%)$. Because of the symmetry at the transition state, both of the weights are exactly the same.

Figure 2 shows the potential energy curve along the reaction path, $R$. As is well known, the reaction proceed via precursor complex in the vicinity of the transition state and the classical double well energy curve is obtained. The changes in the weights of major resonance structures (1, 2 and 3 ) along the reaction are shown in Figure 3. As the reaction proceeds, the weight of $\mathbf{1}$ decreases while that of $\mathbf{3}$ increases. Needless to say, 
Table 3: The weights (\%) of resonance structure in trans-butadiene.

\begin{tabular}{|c|c|c|c|c|c|c|c|}
\hline \multirow[t]{2}{*}{ No. } & & \multirow[t]{2}{*}{ Degeneracy } & \multirow{2}{*}{$\begin{array}{l}\text { Hiberty }^{\mathrm{a})} \\
\text { STO-3G }\end{array}$} & \multicolumn{4}{|c|}{ The present method } \\
\hline & & & & STO-3G & DZV & DZP & TZP \\
\hline 1 & $\mathrm{C}=\mathrm{C}-\mathrm{C}=\mathrm{C}$ & 1 & 22.1 & 23.2 & 23.5 & 22.9 & 22.9 \\
\hline 2 & $\mathrm{C}-\mathrm{C}=\mathrm{C}-\mathrm{C}$ & 1 & 1.0 & 1.8 & 2.2 & 2.0 & 2.0 \\
\hline 3 & $\mathrm{C}^{+}-\mathrm{C}^{-}-\mathrm{C}=\mathrm{C}$ & 2 & 22.6 & 22.8 & 22.7 & 21.8 & 21.8 \\
\hline 4 & $\mathrm{C}^{-}-\mathrm{C}^{+}-\mathrm{C}=\mathrm{C}$ & 2 & 23.2 & 23.6 & 24.3 & 23.8 & 24.0 \\
\hline 5 & $\mathrm{C}^{-}-\mathrm{C}=\mathrm{C}-\mathrm{C}^{+}$ & 2 & 2.1 & 1.7 & 1.9 & 1.9 & 1.9 \\
\hline 6 & $\mathrm{C}-\mathrm{C}^{+}-\mathrm{C}^{-}-\mathrm{C}$ & 2 & 2.1 & 1.7 & 2.0 & 1.8 & 1.8 \\
\hline 7 & $\mathrm{C}^{+}-\mathrm{C}^{-}-\mathrm{C}^{+}-\mathrm{C}^{-}$ & 2 & 12.5 & 12.5 & 12.8 & 12.4 & 12.4 \\
\hline 8 & $\mathrm{C}^{-}-\mathrm{C}^{+}-\mathrm{C}^{+}-\mathrm{C}^{-}$ & 1 & 5.4 & 5.6 & 5.7 & 5.7 & 5.8 \\
\hline 9 & $\mathrm{C}^{+}-\mathrm{C}^{-}-\mathrm{C}^{-}-\mathrm{C}^{+}$ & 1 & 5.1 & 5.2 & 5.0 & 4.8 & 4.8 \\
\hline
\end{tabular}

a) Ref. [45]

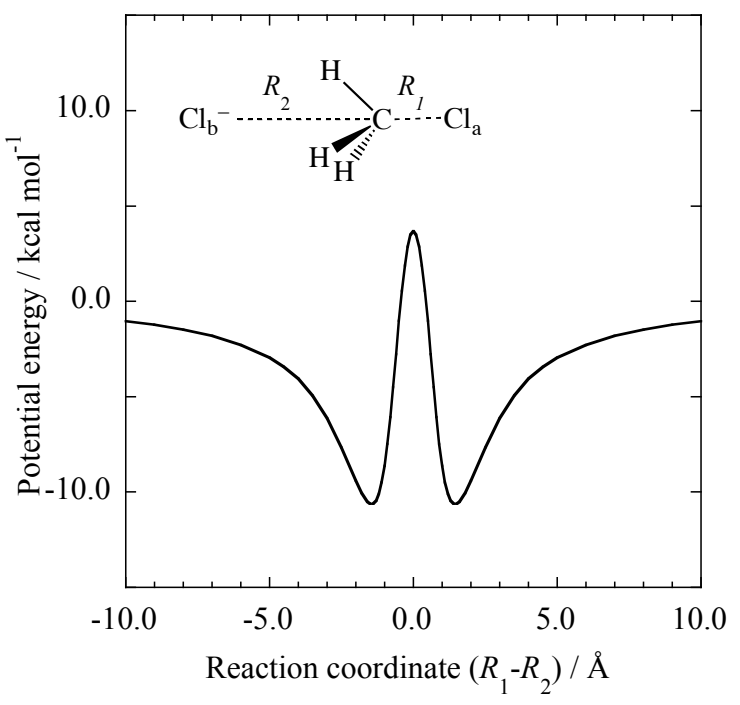

Figure 2: The potential energy curve of the reaction $\mathrm{CH}_{3} \mathrm{Cl}+\mathrm{Cl}^{-} \rightarrow \mathrm{Cl}^{-}+$ $\mathrm{CH}_{3} \mathrm{Cl}$. The reaction coordinate was chosen as $R_{1}-R_{2}$.

Table 4: The weights $(\%)$ of resonance structure in transition state of $\mathrm{S}_{\mathrm{N}} 2$ reaction. $^{\text {a) }}$

\begin{tabular}{ccccr}
\hline \hline No. & & & & Weight \\
\hline $\mathbf{1}$ & $\mathrm{Cl}_{b}^{-}$ & $\mathrm{CH}_{3}$ & $-\mathrm{Cl}_{a}$ & 18.5 \\
$\mathbf{2}$ & $\mathrm{Cl}_{b}^{-}$ & $\mathrm{CH}_{3}^{+}$ & $\mathrm{Cl}_{a}^{-}$ & 63.0 \\
$\mathbf{3}$ & $\mathrm{Cl}_{b}$ & $-\mathrm{CH}_{3}$ & $\mathrm{Cl}_{a}^{-}$ & 18.5 \\
$\mathbf{4}$ & ${ }^{\circ} \mathrm{Cl}_{b}$ & $\mathrm{CH}_{3}^{-}$ & $\mathrm{Cl}_{a}^{\odot}$ & 2.3 \\
$\mathbf{5}$ & $\mathrm{Cl}_{b}^{-}$ & $\mathrm{CH}_{3}^{-}$ & $\mathrm{Cl}_{a}^{+}$ & 1.4 \\
$\mathbf{6}$ & $\mathrm{Cl}_{b}^{+}$ & $\mathrm{CH}_{3}^{-}$ & $\mathrm{Cl}_{a}^{-}$ & 1.4 \\
\hline \hline
\end{tabular}

a) Note that all the sum is greater than $100 \%$ since there are other contributions with small negative values, which appear in Mulliken-type operator.[40] these changes correspond to the bond breaking of $\mathrm{C}-\mathrm{Cl}_{a}$ and new bond formation of $\mathrm{Cl}_{b}-\mathrm{C}$, respectively. The totally ionic structure (2) monotonically increases by the transition state and then decreases after passing through the transition state. The monotonic change in all the weights, even at the precursor complex, implies that the electronic structure in the system continuously changes along the reaction. The stabilization of the precursor complex is simply due to the ion-dipole interaction between $\mathrm{C}-\mathrm{Cl}_{a}$ bond and $\mathrm{Cl}_{b}{ }^{-}$. Numerous studies on this re-

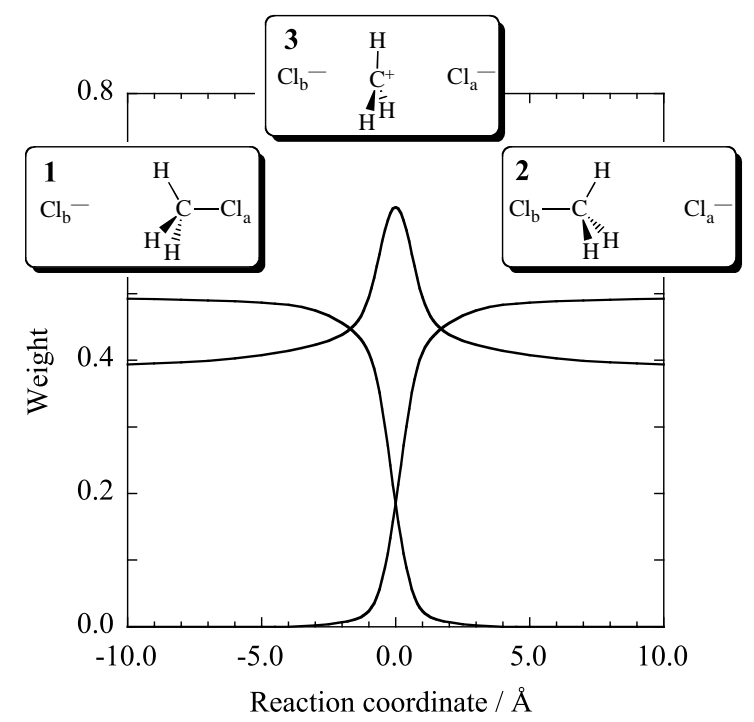

Figure 3: The weights of resonance structure in $\mathrm{CH}_{3} \mathrm{Cl}+\mathrm{Cl}^{-} \rightarrow \mathrm{Cl}^{-}+\mathrm{CH}_{3} \mathrm{Cl}$.

action has been published so far. In particular, Hwang et al. reported the reaction in aqueous solution based on empirical valence bond method,[48] and Mo et al. also reported the same reaction based on molecular orbital-valence bond method.[49] Although both of them mainly discussed the contributions of valence bond structures in terms of energy, the present result is consistent with these works. It should be important to point out that they assumed the three contributions (1, 2 and 3). As 
shown in Table 4, the sum of them is almost $100 \%$, indicating that the description of these three VB contributions is almost equivalent to MO (Hartree-Fock) treatment.

\section{Conclusions}

In this report, we presented a novel method to calculate the weights of resonance structures from HF wave function of four electron system. The method is an extension of our previous one, being consistent with MPA and BOA . Only standard localization procedure is required to obtain the resonance weights. In addition, obtained results agreed well with our chemical intuition. The method is successfully applied to $\mathrm{LiH}, \mathrm{H}_{2} \mathrm{O}$, butadiene and $S_{N} 2$ reaction.

Resonance structure (or valence bond type description) is helpful to understand the nature of chemical bond. The present method is very easy-to-use and provides precise view of the molecular orbitals through a projection based on the second quantization. On the other hand, the method is still limited to closed-shell RHF method, in which the back ground of the spin-flip operator is not clear enough. We still do not have a full picture of this approach, and much more elaboration is highly desired.

\section{Acknowledgements}

This work was financially supported by the Grant-in Aids (Nos. 430-18031019, 19350010, 452-20031014 and 461), all supported by the Ministry of Education, Culture, Sports, Science and Technology (MEXT) Japan.

\section{References}

\section{References}

[1] L. Pauling, The chemical bond, Cornell University Press, Ithaca, NY, 1967.

[2] P.C. Hiberty, C. Leforestier, J. Am. Chem. Soc. 100 (1978) 2012.

[3] E.D. Glendening, F. Weinhold, J. Comput. Chem. 19 (1998) 593.

[4] P. Karafiloglou, J. Comput. Chem. 22 (2001) 306.

[5] P. Karafiloglou, P. Papanikolaou, Chem. Phys. Lett. 342 (2007) 288.

[6] P. Papanikolaou, P. Karafiloglou, J. Phys. Chem. A 112 (2008) 8839.

[7] P. Karafiloglou, J. Chem. Phys. 130 (2009) 164103.

[8] S. Shaik, A. Shurki, Angew. Chem. Int. Ed. 38 (1999) 586.

[9] J.J.W. McDouall, M.A. Robb, Chem. Phys. Lett. 132 (1986) 319.

[10] K. Hirao, H. Nakano, K. Nakayama, M. Dupuis, J. Chem. Phys. 105 (1996) 9227.

[11] Y. Mo, J. Gao, J. Phys. Chem. A 104 (2000) 3012.

[12] Y. Mo, J. Gao, J. Comput. Chem. 21 (2000) 1458.

[13] Y. Mo, J. Gao, J. Phys. Chem. B 107 (2003) 1664.

[14] L. C. Song, J. Gao, J. Phys. Chem. A 112 (2008) 12925.

[15] H. Nakai, Chem. Phys. Lett. 363 (2002) 73.

[16] Y. Kawamura, H. Nakai, H. J. Comput. Chem. 25 (2004) 1882.

[17] Y. Yamauchi, H. Nakai, H. J. Chem. Phys. 123 (2005) 034101.

[18] H. Nakai, Y. Kikuchi, J. Theor. Comput. Chem. 4 (2005) 317.

[19] T. Baba, M. Takeuchi, H. Nakai, Chem. Phys. Lett. 424 (2006) 193.

[20] Y. Imamura, A. Takahashi, H. Nakai, J. Chem. Phys. 126 (2007) 034103.

[21] H. Nakai, Y. Kurabayashi, M. Katouda, T. Atsumi, Chem. Phys. Lett. 438 (2007) 132.

[22] M. Kobayashi, Y. Imamura, H. Nakai, J. Chem. Phys. 127 (2007) 074103.

[23] Y. Imamura, H. Nakai, J. Comput. Chem. 29 (2008) 1555.

[24] Y. Imamura, T. Baba, H. Nakai, Int. J. Quantum Chem. 108 (2008) 1316.
[25] I. Mayer, Chem. Phys. Lett. 97 (1983) 270.

[26] I. Mayer, Int. J. Quantum Chem. 23 (1983) 341.

[27] M. Giambiagi, M.S. de Giambiagi, D.R. Grempel, C.D. Heymann, J. Chim. Phys. 72 (1975) 15

[28] M.S. de Giambiagi, M. Giambiagi, F.E. Jorge, Theor. Chim. Acta 68 (1985) 337

[29] K.C. Mundim, M. Giambiagi, M.S. de Giambiagi, J. Phys. Chem. 98 (1994) 6118.

[30] T. Okada, T. Fueno, Bull. Chem. Soc. Jpn. 48 (1975) 2025.

[31] T. Okada, T. Fueno, Bull. Chem. Soc. Jpn. 49 (1976) 1524.

[32] K.A. Wiberg, Tetrahedron 24 (1966) 1083.

[33] A.E. Reed, F. Weinhold, J. Chem. Phys. 83 (1985) 1736.

[34] P. Pitanga, M. Giambiagi, M.S. De Giambiagi, Chem. Phys. Lett. 128 (1986) 411.

[35] T. Yamasaki, W.A. Goddard, J. Phys. Chem. A 102 (1998) 2919.

[36] T. Yamasaki, D.T. Mainz, W.A. Goddard, J. Phys. Chem. A 104 (2000) 2221.

[37] A. Ikeda, Y. Nakao, H. Sato, S. Sakaki, J. Phys. Chem. A 110 (2006) 9028.

[38] A. Ikeda, D. Yokogawa, H. Sato, S. Sakaki, Chem. Phys. Lett. 424 (2006) 499.

[39] A. Ikeda, D. Yokogawa, H. Sato, S. Sakaki, Int. J. Quantum Chem. 107 (2007) 3132.

[40] A. Ikeda, Y. Nakao, H. Sato, S. Sakaki, J. Chem. Theor. Comp. 5 (2009) 1741.

[41] A.E. Clark, E.R. Davidson, J. Chem. Phys. 115 (2001) 7382.

[42] A.E. Clark, E.R. Davidson, Mol. Phys. 100 (2002) 373.

[43] A.E. Clark, E.R. Davidson, J. Phys. Chem. A 106 (2002) 6890.

[44] I. Mayer, Chem. Phys. Lett. 97 (1983) 270.

[45] P.C. Hiberty, G. Ohanessian, Int. J. Quantum Chem. 27 (1985) 245.

[46] M.W. Schmidt, K.K. Baldridge, J.A. Boatz, S.T. Elbert, M.S. Gordon, J.H. Jensen, S. Koseki, N. Matsunaga, K.A. Nguyen, S.J. Su, T.L. Windus, M. Dupuis, J.A. Montgomery, J. Comput. Chem. 14 (1993) 1347.

[47] J. Pipek, P.G. Mezey, J. Chem. Phys. 90 (1989) 4916.

[48] J.-K. Hwang, G. King, S. Creighton, A. Warshel, J. Am. Chem. Soc. 110 (1988) 5297.

[49] Y. Mo, J. Gao, J. Comput. Chem. 21 (2000) 1458. 